\title{
Ecología y conservación de los bosques y arbustales de Polylepis: ¿qué sabemos y qué ignoramos?
}

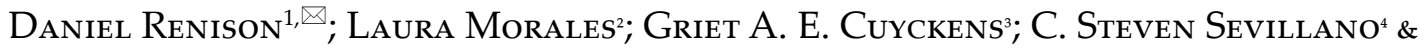 \\ Diego M. Cabrera Amaya \\ ${ }^{1}$ Instituto de Investigaciones Biológicas y Tecnológicas, Centro de Ecología y Recursos Naturales Renovables. CONICET \\ - Universidad Nacional de Córdoba. Córdoba, Argentina. ${ }^{2}$ Department of Plant Sciences and Graduate Group in Ecology, \\ University of California-Davis. California, EE.UU. ${ }^{3}$ Centro de Estudios Territoriales Ambientales y Sociales (CETAS). \\ CONICET - Universidad Nacional de Jujuy. San Salvador de Jujuy, Argentina. ${ }^{4}$ Cornell Lab of Ornithology y Department of \\ Natural Resources, Cornell University. New York, EE.UU. ${ }_{5}^{5}$ Jardín Botánico de Bogotá José Celestino Mutis. Bogotá, Colombia.
}

\begin{abstract}
Resumen. Los bosques de Polylepis, endémicos de las montañas de Sudamérica, suelen estar asociados con sitios relativamente inaccesibles como quebradas, roquedales o laderas empinadas. Las hipótesis más discutidas que explican esta asociación son: 1) factores abióticos como la humedad, la temperatura o el viento, ó 2) factores antropogénicos como la protección del ramoneo por ganado doméstico, la tala o los fuegos iniciados por el ser humano. Para resumir el estado de avance en el conocimiento, realizamos una revisión cuantitativa sobre los estudios de ecología y conservación de bosques de Polylepis a lo largo de toda su distribución. Identificamos 139 publicaciones relevantes, de las que el 36\% estuvo concentrado en la Argentina. Las publicaciones incluyen 20 de las $\sim 27$ especies existentes, de las cuales las más estudiadas son $P$. australis y $P$. tarapacana, con $45 \mathrm{y}$ 21 publicaciones, respectivamente. En el otro extremo, hay 7 especies no representadas en publicaciones. Las disciplinas más populares fueron la ecología de poblaciones $(35 \%)$ y los estudios sobre biodiversidad y comunidades $(18 \%)$. Muy pocos estudios se repiten de una región a la otra. Los estudios que intentan comprender la contribución relativa de factores abióticos vs. antropogénicos para explicar la asociación de los bosques con lugares relativamente inaccesibles señalan una contribución mayor de los factores antrópicos, aunque todos están concentrados en el extremo sur de la distribución de Polylepis en la Argentina. Sugerimos áreas de vacancia en estudios y posibles mejoras en la producción de nuevas publicaciones y en la red de interacciones entre investigadores interesados en los ecosistemas de Polylepis.
\end{abstract}

[Palabras clave: Sudamérica, Andes, distribución, revisión cuantitativa]

\begin{abstract}
Aвstract. Ecology and conservation of Polylepis forests: what do we know and what do we ignore? Polylepis forests, endemic to the Andes of South America, are generally associated to relatively inaccessible sites such as ravines, rocky or steep slopes. The reasons for this association have been widely debated and could be of abiotic origin such as humidity, temperature or winds, or due to anthropogenic origin such as protection from browsing by domestic cattle, felling or human ignited fires. We performed a quantitative review on the ecology and conservation of Polylepis forests with the objective of summarizing the state of the art in this topic. We identified 139 relevant publications; $36 \%$ were concentrated in Argentina. The publications include 20 of the $\sim 27$ existing species; the most studied were $P$. australis and $P$. tarapacana, with 45 and 21 publications, respectively. In the opposite extreme, there were 7 species without publications. The most popular disciplines were population ecology $(35 \%)$ and studies on biodiversity and communities $(18 \%)$. Very few studies of the same type were repeated from one region to the other. The studies that attempt to quantify the relative contribution of abiotic and anthropogenic factors in restricting forest to inaccessible sites show a stronger contribution of human impact, but are all concentrated in the south of distribution of Polylepis forest in Argentina. We suggest research vacancy areas and possible improvements in the production of new publications and in the network of interactions between researchers interested in Polylepis ecosystems.
\end{abstract}

[Keywords: South America, Andes, distribution, quantitative review]

Editor asociado: Esteban Jobbágy

drenison@conicet.gov.ar 


\section{INTRODUCCIÓN}

Los bosques y arbustales montanos dominados por el género Polylepis, y que de ahora en más llamaremos "bosques de Polylepis", se encuentran a lo largo de los Andes y de montañas contiguas, desde la cordillera de Mérida (Venezuela) hasta las Sierras de los Comechingones, en el centro de la Argentina, e incluyen a todos los países Andinos (Arnal et al. 2014). A nivel mundial, Polylepis tarapacana es la especie de porte arbóreo que llega más alto sobre el nivel del mar. Incluso, se pueden encontrar individuos en altitudes superiores a $5000 \mathrm{~m}$ s. n. m. (Cuyckens et al. 2016). Con $\sim 5400 \mathrm{~km}$ entre los extremos norte y sur, los bosques de Polylepis son una de las comunidades boscosas con mayor extensión latitudinal del mundo. A mayores altitudes sobre el nivel del mar, es típico que la canopia esté dominada sólo por especies del género Polylepis, mientras que en las altitudes más bajas son reemplazados por otros géneros como Weinmannia, Alnus, Clethra, Escallonia, Clusia y Maytenus (Navarro et al. 2010; Renison et al. 2013).

El paisaje típico donde se encuentran los Polylepis consiste en parches de diversos tamaños de bosques, asociados con sitios relativamente inaccesibles como quebradas, roquedales o pendientes abruptas. Estos parches de bosques están inmersos en una matriz de pastizales y zonas arbustivas en sitios algo más planos (Cingolani et al. 2014; Fjeldså and Kessler 2004). El motivo de la asociación de los bosques con los sitios inaccesibles es motivo de una larga controversia, central para entender su ecología y para determinar estrategias de conservación, manejo y restauración. Hay autores que adhieren a una hipótesis de distribución de Polylepis influenciada en particular por factores abióticos. Esta hipótesis enfatiza que las quebradas, roquedales o pendientes abruptas podrían tener mejores condiciones para el crecimiento arbóreo, como protección del viento, de heladas o mayor humedad del suelo que otros tipos de topografías (ver síntesis en Kessler 2002; Rada et al. 2009). Otros autores apoyan una hipótesis antropogénica, que sostiene que las quebradas, roquedales o pendientes abruptas podrían brindar un efecto protector del ramoneo de herbívoros o de los fuegos (ver síntesis en Kessler 2002; Renison et al. 2015). La hipótesis es denominada antropogénica porque si bien los herbívoros existen en la zona desde antes que el ser humano, en tiempos modernos se introdujeron especies de herbívoros domésticos y su cantidad aumentó notablemente debido a los cuidados brindados por el humano (Cingolani et al. 2005). Asimismo, el número de igniciones habría aumentado desde la ocupación humana en América del Sur, entre 10 y 14 mil años atrás, y se presume que contribuyó a reducir la extensión de los bosques y a aumentar la de los pastizales (Urrego et al. 2011; Valencia et al. 2016).

Una síntesis realizada por Kessler (2002) sugiere un fuerte componente antrópico en la asociación de los bosques de Polylepis con sitios más o menos inaccesibles. Sin embargo, los estudios llevados a cabo hasta el momento en que Kessler (2002) elaboró la síntesis fueron todos trabajos no experimentales. Al ser del tipo observacional no resultan adecuados para determinar la contribución relativa de los factores abióticos y antropogénicos, dado que los fuegos y los sitios relativamente inaccesibles para la ganadería también tienen condiciones abióticas diferentes a los sitios más accesibles, en términos relativos. En otras palabras, a campo, los disturbios antrópicos y las condiciones abióticas están alineadas y es muy difícil separar la contribución de uno y otro. Para entender las causas de la distribución hacen falta estudios experimentales que incorporen plantaciones y mediciones comparables, tanto del tipo abióticas como de ramoneo por ganado doméstico, fuegos y otros disturbios de origen antropogénico (Kessler 2002). Además de realizar experimentos manipulativos, según Underwood (1997), también está la posibilidad de aprovechar experimentos naturales o de origen antropogénico no planificados. Por ejemplo, se pueden comparar sitios con condiciones abióticas similares, pero con mucha y poca población humana, o sitios afectados y no afectados por fuegos de origen antropogénico. Si bien la interpretación de este tipo de experimentos no planificados puede ser engorrosa debido a tamaños muestrales dispares entre situaciones o tratamientos aplicados sin control del investigador, estos estudios pueden servir para orientar la elaboración de hipótesis sobre las distintas causas de la distribución de los bosques y comprender su significancia.

Además del fuego y del ramoneo por el ganado doméstico, también se mencionan como amenazas para los bosques de Polylepis a la agricultura, la tala, el reemplazo por especies exóticas, el cambio climático y el desarrollo de infraestructura como caminos, 
urbanizaciones y minería. Debido a estas múltiples amenazas, 13 de las especies de Polylepis son consideradas como vulnerables por la IUCN y se aconseja su conservación y restauración (www.iucnredlist.org ${ }_{\iota}$ a saber: $P$. racemosa, $P$. pauta, $P$. reticulata, $P$. pepei, $P$. rugulosa, $P$. subsericans, $P$. weberbaueri, P. hieronymi, P. crista-galli, P. multijuga, P. lanuginosa, P. microphylla, P. neglecta). Más allá de los árboles y los arbustos que dominan los bosques de Polylepis, numerosas especies acompañantes de estos bosques son vulnerables o se encuentran en peligro de extinción (para aves, ver Sevillano et al. en este número). Por esta razón, estamos perdiendo alguno de los componentes de los bosques sin que todavía hayamos comprendido su incidencia en el funcionamiento del ecosistema.

Consideramos que los científicos podemos contribuir a conservar y a restaurar los bosques de Polylepis a través de generar información básica y aplicada que ayude a entender su ecología, a determinar cuáles son sus principales amenazas, a entender las causas de su distribución y a desarrollar técnicas de restauración de bosques degradados. El objetivo de la presente revisión es señalar cuánto abarca la literatura existente y sus limitaciones sobre el conocimiento de la ecología y conservación de los bosques de Polylepis en todo el rango de distribución y desde el punto de vista histórico, geográfico y académico. Las preguntas de investigación son: 1) cuántas publicaciones hay y cómo se distribuyen las contribuciones entre los países, 2) cuáles son las especies de Polylepis, las regiones y las disciplinas de estudio más y menos representadas en la literatura, y 3) qué avances encontramos en una de las preguntas más relevantes: las causas de la asociación entre bosques y sitios relativamente inaccesibles. Así, intentamos orientar a la comunidad de estudiantes y científicos en cuanto a las regiones, especies y temáticas a investigar en el futuro. Además, con el fin de optimizar los recursos humanos discutimos la pertinencia de modificar, o no, la trama de interacciones que existe entre los científicos dedicados al estudio de los bosques de Polylepis.

\section{Materiales y Métodos}

Para señalar cuánto abarca y cuáles son las limitaciones de la literatura académica en cuanto al conocimiento de la ecología y conservación de los bosques y arbustales de Polylepis realizamos una revisión sistemática de la literatura. Para ello, nos basamos en el método descripto por Pickering y Byrne (2014) y Pickering et al. (2014). Primero, generamos una base de datos de artículos científicos en temas relacionados a la ecología y la conservación de bosques de Polylepis, publicados en revistas indexadas, y realizamos una búsqueda sistemática en cuatro bases de datos: Web of ScienceTM Core Collection (WSCC) (a través de UC Davis University Library), BIOSIS Citation Index (BCI), SciELO Citation Index(SCC), SCOPUS ${ }^{\circ}$ y el portal de Scientific Electronic Library Online (www.scielo.org). En esas bases de datos realizamos una búsqueda inicial en el título, en el resumen o en las palabras clave de los artículos. La búsqueda incluyó la siguiente sintaxis: Polylepis NOT Anolis NOT Norops NOT Dendroaspis. Usamos Polylepis en vez de "Polylepis woodland" o "Polylepis forest" porque estos últimos excluían algunos artículos relevantes. Usamos los operadores NOT para excluir a otros grupos taxonómicos que tienen nombres de especie polylepis y son frecuentes en la literatura científica. Además, para asegurarnos que con los métodos anteriores no estuviéramos obviando alguna publicación citada sobre bosques y arbustales de Polylepis que no tuviera la palabra Polylepis en título, en el resumen o en las palabras clave, buscamos la palabra Polylepis en el portal de SciELO (utilizando el método integrado en los listados regionales) y en Google Académico. En este servicio revisamos los 200 estudios más citados y gracias a ello pudimos agregar una publicación a la base de datos.

Realizamos las búsquedas entre el 24 y el 25 de abril de 2016. Juntamos los registros de las búsquedas iniciales en distintas bases de datos en el programa EndNote7 y removimos registros de títulos duplicados automáticamente. El resultado fue una base de datos de 305 artículos publicados en revistas indexadas. Mediante lectura de los títulos, resúmenes y, en ocasiones, todo el artículo, descartamos 179 artículos para dejar en la revisión sólo artículos originales en los que al menos uno de los focos principales fuera la ecología de los bosques o arbustales de Polylepis (la lista está disponible por correo electrónico al autor para correspondencia). En la revisión no incluimos descripciones taxonómicas, estudios sobre la ecología de especies acompañantes a los Polylepis, observaciones de nuevas distribuciones ni estudios sobre biodiversidad cuyo foco no fueran los bosques de Polylepis. Tampoco incluimos a la dendroclimatología dentro de 
la revisión porque esos trabajos se enfocan en elucidar el clima pasado y, además, Boninsegna et al. (2009) realizaron una revisión sobre el tema. Excluimos capítulos de libro para evitar duplicar la información y no usamos literatura gris (e.g., tesis y artículos en revistas que no se encuentran en las bases de datos mencionadas) dada la dificultad de conseguirla de forma sistemática.

Para cada artículo extrajimos la siguiente información, que sistematizamos en una planilla de cálculo: 1) datos básicos del artículo, como autores y sus afiliaciones, año de publicación, idioma y revista en la que se publicó, 2) la principal, o las dos principales, disciplina/s de estudio del artículo, 3) el tipo de método utilizado, 4) las especies de Polylepis involucradas en el estudio, 5) las coordenadas geográficas de las áreas de estudio, y 6) anotamos si al menos uno de los objetivos incluía la evaluación simultánea de influencias abióticas y antropogénicas sobre la asociación de los bosques con los sitios inaccesibles.

En los casos de autores con dos o más filiaciones, priorizamos la que estuviera en un país andino. Para obtener las coordenadas geográficas usamos las provistas en el texto o mapa de la publicación. En el caso de haber sido un área, sólo se indica un punto. Si se abarcaron varios sitios, los incluimos a todos con un punto cada uno. Si esta información no estaba presente, la dedujimos del mapa con la ayuda de Google Earth®. Transformamos todas las coordenadas a grados decimales (WGS84) y sólo utilizamos puntos con, por lo menos, un decimal, y mapeamos los resultados. Las principales disciplinas de estudio las clasificamos en: 1) poblacionales, que abarcaron estructura de edades o tamaños, regeneración, crecimiento y supervivencia de adultos, productividad y viabilidad de las semillas, 2) interacciones bióticas

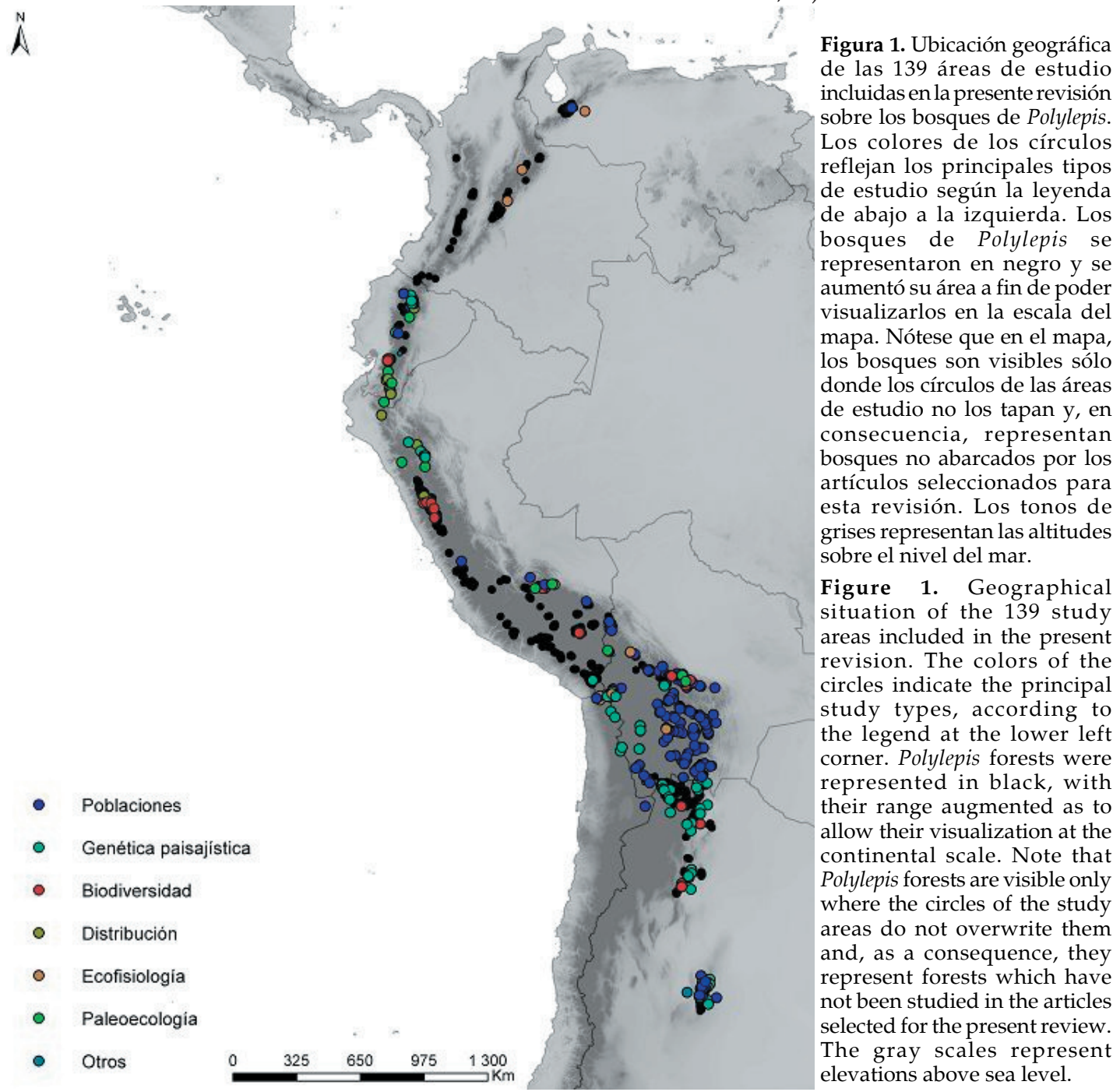


como micorrizas, facilitación, competencia, herbivoría de origen no antropogénico y depredación, 3) biodiversidad y comunidades de plantas, hongos, mamíferos, aves, insectos u otros taxones, 4) paleoecología, 5) genética paisajística, 6) ecofisiología, 7) hidrología, 8) dendroecología, 9) estudios de captura carbono, 10) de distribución, 11) fenología, 12) polinización, y 13) ecología de las semillas. $\mathrm{Si}$ bien incluimos todas estas categorías en el mapa, para más claridad unificamos las menos representadas en "otros": hidrología, dendroecología y estudios de captura de carbono (Figura 1). Intentamos clasificar a las publicaciones por su contribución a la conservación y restauración de los bosques de Polylepis, pero este tipo de clasificación nos resultó muy subjetiva, ya que casi todas tenían texto aludiendo una contribución a dichas temáticas y nos era difícil decidir la importancia real de dicha contribución.

Para determinar qué podemos decir sobre la contribución relativa de los factores abióticos y antropogénicos en la asociación de los bosques de Polylepis con los sitios relativamente inaccesibles identificamos los artículos relevantes mediante lectura completa de todos los artículos. Para que el artículo fuera considerado relevante debía analizar de forma comparable algún factor abiótico relacionado con la inaccesibilidad y, al menos, algún factor de origen antropogénico. Entre los factores abióticos consideramos la pendiente, la topografía, la proporción de roquedales, la fisiografía o la rugosidad del terreno. No consideramos la altitud sobre el nivel del mar (e.g., Toivonen et al. 2011) ya que no necesariamente se relaciona con la accesibilidad y en ningún caso se aclaró que en el sitio de estudio la elevación está asociada con la accesibilidad. Consideramos la posibilidad de que las evaluaciones sobre influencias abióticas y antropogénicas sean reportadas en publicaciones diferentes (e.g., la revisión de Kessler et al. 2002) e inicialmente incluimos estos casos en la revisión; no obstante, en versiones posteriores descartamos esta aproximación dado que no aportaba nada nuevo a la revisión de Kessler (2002) y nos fue imposible realizar aportes acerca de la contribución relativa de los distintos factores cuando eran reportadas en distintos artículos.

\section{Análisis de datos}

Para la mayor parte de los resultados usamos conteos de publicaciones y promedios. Las redes de colaboración están basadas sobre el sistema de nodos-vínculos-nodos (del inglés 'vertex-links'). Definimos red de colaboración como la colaboración dada entre un primer autor y un co-autor que publicaron en conjunto cada artículo. El análisis incluyó un total 274 autores y co-autores (a los que se definió como nodos) que generaron 415 líneas de coautoría (vínculos). Para cada uno de los países donde se distribuye Polylepis (con excepción de Chile, donde no hubo primeros autores de esa nacionalidad), se estimó un índice de colaboración determinado por el cociente entre el número de líneas de colaboración y el número de autores. Un índice más alto indica mayor colaboración por autor y publicación. Este índice no está sopesado por el número de publicaciones (índice de colaboración beta), ya que el número de publicaciones por país se reporta por separado.

\section{Resultados}

Número de publicaciones y países contribuyentes

Identificamos 139 publicaciones relevantes; de éstos, los más antiguos fueron las publicaciones sobre comunidades de plantas en bosques de Polylepis australis del centro de la Argentina (Cabido and Acosta 1985) y sobre ecofisiología de Polylepis sericea en Venezuela (Rada et al. 1985). Tanto Cabido como Rada siguieron publicando artículos hasta el presente y su filiación corresponde a institutos locales de las áreas de estudio. Para los 14 años entre 1986 y 1999 identificamos solamente 8 artículos; en éstos se incorporan ecólogos con filiación institucional en Europa (entre ellos cabe mencionar a Fjeldsa, Hensen y Kessler). A partir del año 2000, el número de artículos aumentó de manera exponencial, con una meseta de 43 artículos cada cuatro años (Figura 2).

En 55\% de las publicaciones (77 de las 139), el primer autor de la publicación poseía una filiación correspondiente a una institución de un país andino (donde hay bosque de Polylepis). Para los países andinos, el número de publicaciones por país de filiación del primer autor fueron: 45 para la Argentina, 14 para Bolivia, 7 para Venezuela, 5 para Perú, 4 para Ecuador y 2 para Colombia. Chile quedó sin representación como país de filiación del primer autor. Para los países no andinos, el número de publicaciones por país de filiación del primer autor fueron: 25 para Alemania, 17 para EE.UU., 8 para el Reino Unido y 9 publicaciones más, repartidas 


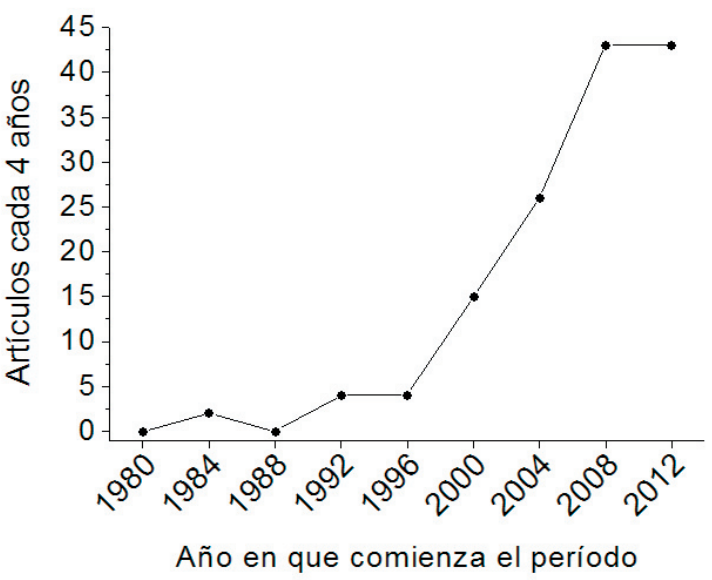

Figura 2. Evolución en el tiempo del número de artículos sobre bosques y arbustales de Polylepis. De los 139 artículos relevantes para el presente estudio, tres publicaciones del 2016 fueron omitidos de este gráfico.

Figure 2. Evolution of the number of Polylepis forest and shrubs studies over the time. From the 139 papers of the present study, we omitted three papers published in 2016.

entre España, Suiza, Dinamarca, Finlandia, Canadá y Checoslovaquia. En 20 de las 62 publicaciones, cuando el primer autor tenía filiación en un país no andino, al menos uno de los co-autores poseía filiación en un país andino. El índice de colaboración elaborado a partir de las redes de co-autorías muestra que Venezuela, Argentina y los países de Norteamérica y Europa tuvieron el mayor grado de colaboraciones entre co-autores,

Tabla 1. Para cada país se muestra el número de las líneas de colaboración entre autores, el número de autores y co-autores involucrados en los cálculos y el índice de colaboración (ver análisis estadísticos). Nótese que el número total de autores no es igual a la sumatoria debido a que algunos autores poseen filiaciones en más de un país.

Table 1. For each country, we show the number of research links, number of authors and co-authors involved in the calculations and the collaboration indexes between authors. Note that the total number of authors does not equal the sum because some authors report affiliations in more than one country.

\begin{tabular}{lccc}
\hline País & $\begin{array}{c}\text { Líneas de } \\
\text { colaboración }\end{array}$ & $\begin{array}{c}\text { Número de } \\
\text { autores }\end{array}$ & $\begin{array}{c}\text { Índice de } \\
\text { colaboración }\end{array}$ \\
\hline Argentina & 152 & 87 & 1.7 \\
Bolivia & 33 & 66 & 0.5 \\
Perú & 12 & 16 & 0.7 \\
Ecuador & 12 & 16 & 0.7 \\
Venezuela & 25 & 13 & 1.9 \\
Colombia & 7 & 7 & 1.0 \\
Norte América & 173 & 126 & 1.4 \\
y Europa & 415 & 274 & \\
\multicolumn{1}{c}{ Total } & 45 & & \\
\hline
\end{tabular}

mientras que Bolivia presentó el menor índice de colaboración (Tabla 1).

\section{Especies de Polylepis, regiones y disciplinas de estudio}

El conjunto de los 139 artículos relevantes abarcó 20 de las 27 especies de Polylepis descriptas en Schmidt-Lebuhn et al. (2006); $P$. australis y $P$. tarapacana fueron las especies más representadas, con 48 y 21 publicaciones, respectivamente. Le siguen, en número decreciente de publicaciones, $P$. sericea (13), P. tomentella (10), P. racemosa (10), P. besseri (8), P. incana (8), P. pauta (7), P. reticulata (5), P. pepei (4), P. rugulosa (4), P. subsericans (3), P. weberbauerii (3), P. quadrijuga (2), P. hieronymi (2), P. lanata (2), P. crista-galli (1), P. incarum (1), P. multijuga (1), P. subtusalbida (1). Ninguna de las 139 publicaciones incluía estudios sobre las 7 especies restantes: P. flavipila, $P$. lanuginosa, P. microphylla, $P$. neglecta, $P$. pacensis, $P$. pallidistigma y $P$. triacontandra. En 16 artículos no se mencionaba la especie de Polylepis; entre ellos, 9 estudios fueron paleoecológicos con polen o carbón, en los que - por el momento - es imposible identificar a la especie. Sin embargo, también hubo 4 estudios de biodiversidad de aves o mamíferos y 3 estudios de distribución realizados con fotografías o sensores remotos. Las áreas de estudio estaban situadas en la Argentina (55 publicaciones), Bolivia (41), Perú (21), Ecuador (19), Chile (8), Venezuela (4) y Colombia (2) (Tabla 2).

Las disciplinas de estudio más populares entre las 139 publicaciones fueron la ecología de poblaciones (49 publicaciones) y los

Tabla 2. Número de publicaciones y porcentaje en función del país donde se encuentran las áreas de estudio $(\mathrm{N}=139$ publicaciones). Nótese que la suma de publicaciones por país es mayor al número de publicaciones debido a las publicaciones que incluyeron áreas de estudio en más de un país.

Table 2. Number of publications and percentage as a function of the country where the study area is situated ( $\mathrm{N}=139$ publications). Note that the sum of the publications per country is higher than the number of publications because of publications which included study areas in more than one country.

\begin{tabular}{lll}
\hline País & $\mathrm{N}$ & $\%$ \\
\hline Argentina & 55 & 37 \\
Bolivia & 41 & 27 \\
Perú & 21 & 14 \\
Ecuador & 19 & 13 \\
Chile & 8 & 5 \\
Venezuela & 4 & 3 \\
Colombia & 2 & 1 \\
\hline
\end{tabular}


estudios de biodiversidad y comunidades (25). Siguen las publicaciones de ecofisiología (15), paleoecología (15), genética paisajística (13), distribución (11) y sobre interacciones bióticas (8). Menos representados estuvieron los estudios relacionados con la hidrología (2) y los suelos (2). Por otro lado, las disciplinas de estudio no estaban repartidas equitativamente entre los países. Por ejemplo, los estudios poblacionales estuvieron concentrados en la Argentina y Bolivia (25 y 11, respectivamente, de un total de 49); los estudios de biodiversidad y comunidades se repartieron casi todos entre la Argentina, Bolivia y Perú $(8,8$ y 6 , respectivamente, de un total de 25) y los estudios ecofisiológicos se repartieron entre Bolivia, Venezuela y Colombia (5, 3 y 3, respectivamente, de un total de 15). La mayoría de los trabajos fueron de carácter observacional (77\%), mientras que los experimentales, de modelamiento o combinados representaron el restante 33\%. De los trabajos evaluados pudimos obtener las coordenadas geográficas sólo en 119 publicaciones; incluyeron 430 sitios de estudio:
44 de biodiversidad, 18 de distribución, 15 de ecofisiología, 105 de genética paisajística, 14 de paleoecología, 221 de poblaciones y 13 "otros" (Figura 1). Las publicaciones incluyeron entre 1 y 221 sitios de estudio (Kessler et al. 2007). De 20 artículos no pudimos obtener coordenadas geográficas.

\section{El ejemplo de las causas de la distribución}

La contribución relativa de la influencia de los factores abióticos y antropogénicos a la asociación de los bosques con los sitios relativamente inaccesibles fue evaluada en 10 publicaciones (Tabla 3). En 9 publicaciones se usó una aproximación comparativa entre situaciones con distintas condiciones abióticas y disturbios de origen antropogénico, mientras que en una de las publicaciones (Renison et al. 2015) se usó una aproximación completamente experimental, con siembras y plantaciones, como recomendó Kessler (2002). Sin embargo, las 10 publicaciones tienen una representación geográfica relativamente restringida y sólo involucran a P. australis, ubicada en la porción

Tabla 3. Estudios comparativos y experimentales que intentan determinar la contribución relativa de la influencia de los factores abióticos y antropogénicos a la asociación de los bosques de Polylepis con los sitios relativamente inaccesibles. Se muestra la cita de la publicación, los factores abióticos y de origen antropogénico que se reportan en el estudio como influyentes en los bosques, el signo de la influencia (entre paréntesis) y la variable de respuesta considerada en el estudio.

Table 3. Comparative and experimental studies performed to determine the relative contribution of abiotic and anthropogenic factors which forge the association of Polylepis forests to relatively inaccessible sites. We show the citation of the publication, the abiotic and anthropogenic factors that were considered, the sign of the influence (in parenthesis) and the response variable considered.

\begin{tabular}{|c|c|c|c|}
\hline $\begin{array}{l}\text { Estudios } \\
\text { Comparativos }\end{array}$ & Factores abióticos & Factores antropogénicos & Variable de respuesta \\
\hline Renison et al. (2002) & $(+)$ roca & (-) quema & $\begin{array}{l}\text { Supervivencia y crecimiento de } \\
\text { juveniles y adultos }\end{array}$ \\
\hline Renison et al. (2004) & (-) roca & (-) erosión de suelos & Viabilidad de semillas \\
\hline Renison et al. (2006) & $\begin{array}{l}\text { (ns) topografía } \\
\text { (ns) roca }\end{array}$ & (-) índice de perturbación & Cobertura de Polylepis \\
\hline Cingolani et al. (2008) & $\begin{array}{l}(+) \text { pendiente } \\
(+) \text { topografía }\end{array}$ & (-) proximidad a casas y caminos & $\begin{array}{l}\text { Presencia de unidades } \\
\text { vegetales con Polylepis }\end{array}$ \\
\hline Renison et al. (2011) & (ns) topografía & $\begin{array}{l}\text { (-) perturbación general (erosión, } \\
\text { ganadería, número de casas) }\end{array}$ & $\begin{array}{l}\text { Complejidad estructural del } \\
\text { bosque }\end{array}$ \\
\hline Torres et al. (2008) & (ns) pendiente & (-) ganadería y erosión de suelos & $\begin{array}{l}\text { Densidad de plántulas- } \\
\text { regeneración }\end{array}$ \\
\hline Zimmerman et al. (2009) & (ns) pendiente & (ก) ganadería & $\begin{array}{l}\text { Regeneración (presencia/ } \\
\text { ausencia de plántulas) }\end{array}$ \\
\hline Pollice et al. (2013) & $\begin{array}{l}\text { (ns) roca } \\
\text { (ns) pendiente }\end{array}$ & (-) ganadería & Producción de semillas \\
\hline $\begin{array}{l}\text { Alinari et al. (2015) } \\
\text { Experimental }\end{array}$ & $(+)$ topografía & $\begin{array}{l}\text { (-) quema } \\
\text { (-) ganadería }\end{array}$ & $\begin{array}{l}\text { Supervivencia y crecimiento de } \\
\text { adultos }\end{array}$ \\
\hline Renison et al. (2015) & $(+)$ topografía & (-) ganadería & $\begin{array}{l}\text { Establecimiento temprano, } \\
\text { supervivencia y crecimiento de } \\
\text { juveniles }\end{array}$ \\
\hline
\end{tabular}


más austral de la distribución del género en el centro de la Argentina.

Los autores del estudio comparativo y más amplio usaron un sistema de información geográfica y regresiones logísticas multinomiales para determinar la probabilidad de presencia de Polylepis en función de la distancia a casas y caminos, asumiendo que a mayor distancia de casas y caminos, menor era el impacto del ser humano, entre otras variables explicativas (Tabla 3) (Cingolani et al. 2008). Así, los autores reportaron mayor cantidad de bosques y matorrales a mayores distancias de casas y caminos, aunque también encontraron una asociación con posiciones topográficas bajas, mayores pendientes y fisiografías escarpadas, entre otras variables abióticas. En la actualidad, los bosques y matorrales de Polylepis ocupan $12 \%$ del paisaje estudiado. Las modelaciones que simulan un paisaje alejado de casas y caminos arribaron a un valor de $48 \%$ de la superficie cubierta por bosques y matorrales de Polylepis (Cingolani et al. 2008). Ello sugiere que si se reduce la influencia humana a los niveles encontrados en los sitios más alejados de casas y caminos, los bosques aumentarían tres veces su superficie actual. Casi todos los estudios restantes sugieren efectos negativos de la ganadería o de los fuegos sobre algún estadio del ciclo de los árboles o sobre su densidad o sobre la estructura del bosque (Tabla 3). La excepción sería el estudio de Zimmerman et al. (2009), que para la ganadería señala mayor presencia de plántulas a densidades ganaderas intermedias debido a las dificultades en el establecimiento en sitios con más de 55\% de cobertura de broza, típico de los sitios con exclusión ganadera completa. Para las variables abióticas generalmente asociadas a sitios inaccesibles, los estudios no señalan ningún efecto en 6 de las 9 publicaciones que reportan estudios comparativos, un efecto negativo de la roca sobre la viabilidad de las semillas, un efecto positivo de la roca sobre la protección de los fuegos, y menor daño por fuego y mayor regeneración post-fuego en una quebrada inaccesible que en una cumbre más accesible (Tabla 3).

El único estudio experimental involucró siembras y plantaciones en tres posiciones topográficas y dos situaciones de carga ganadera sin repeticiones y que integró 17 años de monitoreo. Para un indicador de la biomasa producida por siembra o plantación, los autores encontraron que la topografía era
5 veces más importante en la regeneración temprana (siembra y monitoreo de 5 años), pero que la ganadería se torna importante en aumentar la mortalidad y reducir el crecimiento de juveniles (plantación y monitoreo de 12 años). En este sentido, la ganadería llega a ser 5.4 veces más importante que la topografía cuando se integran los resultados en 17 años de monitoreo mediante multiplicación de matrices (Renison et al. 2015). Este resultado se debe principalmente al ramoneo cada vez más intenso que sufren los arbolitos mayores a $5 \mathrm{~cm}$ de altura a medida que crecen.

\section{Discusión}

\section{Número de publicaciones, afiliación y red de interacciones entre autores}

El número de publicaciones sobre ecología y conservación de bosques y arbustales de Polylepis fue relativamente alto si lo comparamos con otro género andino y estrictamente neotropical como Escallonia. Aquí, una búsqueda similar a la realizada para Polylepis brinda 5 veces menos publicaciones. Sin embargo, una búsqueda similar para Nothofagus, Quercus, Eucalyptus y Pinus genera 9, 76, 62 y 176 veces más publicaciones, respectivamente, que para Polylepis (datos no reportados). Sobre la base de estas comparaciones, creemos que aún queda mucho por investigar y publicar sobre los bosques de Polylepis y sugerimos que sería adecuado aumentar el número de publicaciones existentes en, al menos, un orden de magnitud en los próximos 10 años. De esa manera podríamos acercarnos o igualar lo que ahora hay publicado sobre Nothofagus, y así realizar aportes y revisiones relevantes acerca de aspectos genéticos, poblacionales, de biodiversidad, manejo y conservación (e.g., Gea-Izquierdo et al. 2004; Lavandero et al. 2009; Premoli et al. 2012). Sin embargo, es preocupante que la tasa de publicaciones sobre bosques y arbustales de Polylepis no haya seguido aumentando en los últimos 4 años analizados en el presente estudio. Se requerirá un gran esfuerzo para alcanzar esta meta.

Para aumentar el número de publicaciones se necesitarán más recursos, además de optimizar los existentes. Consideramos que un aumento de recursos (e.g., sueldos para investigadoresdocentes, subsidios e infraestructura) podría contribuir a lo que sabemos de los bosques de Polylepis y al número de publicaciones, 
en especial en los países andinos, que están más rezagados en cuanto a financiamiento en ciencia y tecnología. Otro aspecto a considerar es optimizar los recursos existentes. A modo de ejemplo, al momento de elaborar la presente discusión (diciembre de 2016), de un total de 156 resúmenes presentados a los congresos de Ecología y Conservación de Bosques de Polylepis entre 2006 y 2013 sólo encontramos que $13.5 \%$ fueron publicados y están reflejados en alguna de las 139 publicaciones de esta revisión (datos no reportados). Con el fin de promover la publicación de las investigaciones, creemos que es necesario aumentar y consolidar redes de colaboración entre científicos a lo largo de Latinoamérica (Sancho et al. 2006). En particular, pueden ser fundamentales las redes que vinculen a científicos con capacidad para la colectar y analizar datos con investigadores con práctica y formación en el proceso de escribir las publicaciones. A largo plazo, también se optimizarían los recursos si se busca estandarizar el diseño de muestreo y los métodos de medición lo más posible. De este modo se podrían encontrar patrones comunes y responder interrogantes a diferentes escalas espaciales de forma más simple y eficiente. Para ello, instamos a repetir diseños y métodos ya usados en estudios de Polylepis o, eventualmente, adherirse a protocolos ya existentes, con las adaptaciones necesarias en cada caso.

Finalmente, dado que la conservación y la restauración de los bosques es más efectiva cuando involucra a actores locales, consideramos que es saludable que haya una proporción relativamente alta de científicos radicados en institutos de investigación cercanos a los bosques de Polylepis. Los científicos y otros actores locales pueden tener mayor interés y conocimiento local de la problemática y del comportamiento biológico, como así también de las normativas e instituciones sociales de la zona. Al estar presentes localmente, los estudios pueden tener un seguimiento más cercano y de menor costo. También consideramos saludable que los investigadores de las distintas regiones interactúen entre ellos y, en cierta proporción, con investigadores no locales, capaces de brindar una visión y un entendimiento más global de las preguntas a responder en las investigaciones. En consecuencia, instamos a los investigadores no locales a conseguir socios locales e involucrarlos como co-autores de las publicaciones.
Especies de Polylepis, regiones y disciplinas de estudio

Sobre la base de este trabajo, consideramos que aún faltan realizar muchos estudios en bosques de Polylepis. Esto es verdad para todas las especies, regiones y temáticas. Además, nuestros resultados sugieren algunas prioridades, incluyendo las especies subrepresentadas en número de publicaciones (ver resultados). También tendrían prioridad las áreas de conocimiento y las regiones poco estudiadas, como los estudios eco-fisiológicos en la Argentina, Perú, Chile y Ecuador, y los estudios poblacionales en regiones como Colombia y el centro y norte de Perú (Figura 1).

Consideramos, además, que sería positivo investigar en detalle un mínimo de tres a cinco especies de Polylepis situadas en regiones con climas contrastantes. Así, la ecología, la fisiología y la genética de cada especie estaría representada con 100 a 200 publicaciones y se podrían obtener conclusiones sólidas sobre estas especies, extrapolables a otras especies de Polylepis. Afirmamos lo antedicho porque los bosques de Polylepis son ecosistemas únicos que ocurren en un amplio rango altitudinal, extensión latitudinal y diversidad de climas. Casi no existen otras regiones similares en el mundo que estén bien estudiadas y de las cuales podamos aprovechar lecciones aprendidas, ya que otras zonas de montañas tropicales y subtropicales están restringidas a África y Asía, donde también existen escasas publicaciones sobre los bosques de altura.

\section{El ejemplo de las causas de la distribución}

Los estudios revisados aquí asocian mayor actividad humana con algún grado de reducción de presencia, densidad, o desempeño de Polylepis, y sugieren que la asociación de bosques con sitios inaccesibles es principalmente por disturbios humanos. Tampoco descartan una influencia menor de factores abióticos, lo que concuerda con las conclusiones de la revisión de Kessler (2002), realizada con estudios observacionales y que noincluían simultáneamente a efectos abióticos y antropogénicos. Dicho esto, la inferencia que podemos hacer en cuanto a la extensión geográfica de los estudios es pequeña, ya que todos estaban centrados en la distribución sur de los bosques donde encontramos únicamente a Polylepis australis. Consideramos que para encontrar patrones, estos estudios híbridos en los que se estudian factores abióticos asociados 
a la inaccesibilidad y disturbios humanos resultarán fundamentales en la distribución centro y norte de los bosques de Polylepis. Se debe destacar que los bosques de Polylepis están íntegramente dentro de los climas que Whittaker (1975) denominó ecosistemas inciertos. En estos climas, el balance entre tipos de vegetación leñosa y herbácea puede estar influenciado hasta cierto punto por los grandes herbívoros y también por los fuegos (Bond 2005). Entonces, podemos sospechar que la herbivoría por ganado doméstico y los fuegos antropogénicos pueden estar influenciando la distribución de los bosques de Polylepis en otros sitios no estudiados. Esto lo discutió Kessler (2002) sobre la base de estudios con distribución geográfica más amplia, pero que no contemplaban factores abióticos relacionados con la inaccesibilidad y antropogénicos en el mismo trabajo. Como resumen de esta sección, luego de una década y media, hacemos nuestra y reiteramos la opinión de Kessler (2002), que sostiene que necesitamos hacer estudios híbridos que repitan manipulaciones experimentales a través de distintos gradientes de influencias abióticas o topográficas. Además, los estudios observacionales diseñados con cuidado (e.g., con muestreo estratificado, selección de sitios con ayuda de herramientas SIG, etc.) para cubrir más áreas y especies aumentarán el poder analítico de este tipo de estudios. Los modelos de distribución de especies que incorporen formas de cuantificar el efecto de distintos factores ambientales también podrían ser una herramienta para entender mejor los factores que determinan las distribuciones de Polylepis (Elith and Leathwick 2009). Sin embargo, los modelos son hipótesis que deben ser comprobadas con estudios de campo para descubrir la importancia de los distintos tipos de variables.
Dado que la temática de la distribución de los bosques a los sitios más inaccesibles es una de las preguntas más estudiadas, cabe esperar que debiera ser más difícil aún realizar inferencias geográficamente amplias sobre otras preguntas menos abordadas. Estas preguntas incluyen temáticas importantes como: ¿cuáles son efectos de la fragmentación sobre la diversidad genética en las distintas especies de Polylepis? O bien, ¿por qué es tan baja la viabilidad de las semillas de Polylepis en las especies estudiadas hasta el momento?

\section{Conclusiones}

La comunidad de científicos que estudian a los bosques de Polylepis y la "Polylepología" se encuentra relativamente bien establecida. Con el fin de poder hacer inferencias sólidas sobre aspectos ecológicos de estos bosques en base a los estudios científicos, a futuro queda una etapa de crecimiento en número de científicos, número de publicaciones, formación de redes y realización de estudios similares en las distintas regiones andinas. De esta manera podremos comenzar a entender la ecología de estos bosques y a realizar aportes sólidos a su conservación y restauración. Mientras tanto, existe una base de más de 100 publicaciones que representa un aporte significativo para la toma de decisiones de manejo.

Agradecimientos. A los sucesivos organizadores de los congresos de Ecología y Conservación de Bosques de Polylepis, quienes, en cuatro ocasiones, permitieron madurar la idea de realizar el presente estudio. A CONICET-DFG por proveer a DR una parte de los fondos necesarios para asistir al IV congreso de la especialidad.

\section{REFERENCIAS}

Alinari, J., A. von Muller, and D. Renison. 2015. The contribution of fire damage to restricting high mountain Polylepis australis forests to ravines: insights from an un-replicated comparison. Ecología Austral 25:11-18.

Arnal, H., A. Sampson, G. Navarro, W. Palomino, W. Ferreira, K. Romoleroux, D. Caro, I. Teich, E. Cuyckens, C. Antezana, S. Arrazola, C. Aucca, J. Balderrama, S. Beck, S. Burneo, N. De la Barra, Y. Fandinso Ferro, G., I. Gómez, G. Guzmán, J. Iglesias, J. Irazabal, P. Lozano, M. Mercado, A. Monsalve, D. Renison, S. Salgado, and E. Samochuallpa. 2014. Mapa pan Andino de bosques de Polylepis prioritarios para conservación. The Plains, USA.

Bond, W. J. 2005. Large parts of the world are brown or black: A different view on the "Green World" hypothesis. Journal of Vegetation Science 16:261-266.

Boninsegna, J. A., J. Argollo, J. C. Aravena, J. Barichivich, D. Christie, M. E. Ferrero, A. Lara, C. Le Quesne, B. H. Luckman, M. Masiokas, M. Morales, J. M. Oliveira, F. Roig, A. Srur, and R. Villalba. 2009. Dendroclimatological reconstructions in South America: A review. Palaeogeography, Palaeoclimatology, Palaeoecology 281:210-228.

Cabido, M., and A. Acosta. 1985. Estudio fitosociológico en bosques de Polylepis australis BITT. ("Tabaquillo") en las Sierras de Córdoba, Argentina. Documents Phytosociologiques 9:385-400.

Cingolani, A., D. Renison, P. Tecco, D. E. Gurvich, and M. Cabido. 2008. Predicting cover types in a mountain range with long evolutionary grazing history: a GIS approach. J Biogeogr 35:538-51.

Cingolani, A. M., M. V. Vaieretti, M. A. Giorgis, M. Poca, P. A. Tecco, and D. E. Gurvich. 2014. Can livestock grazing 
maintain landscape diversity and stability in an ecosystem that evolved with wild herbivores? Perspectives in Plant Ecology, Evolution and Systematics 16:143-153.

Cingolani, A., I. Noy-Meir, and S. Díaz. 2005. Grazing effects on rangeland diversity: diversity-intensity and state and transition models. Ecological Applications 15:757-73.

Cuyckens, G. A. E., D. A. Christie, A. I. Domic, L. R. Malizia, and D. Renison. 2016. Climate change and the distribution and conservation of the world's highest elevation woodlands in the South American Altiplano. Global and Planetary Change 137:79-87.

Elith, J., and J. R. Leathwick 2009. Species distribution models: ecological explanation and prediction across space and time. Annual Review of Ecology, Evolution, and Systematics 40:677-697.

Fjeldså, J., and M. Kessler. 2004. Conservación de la biodiversidad de los bosques de Polylepis de las tierras altas de Bolivia. Centro para la Investigación de la Diversidad Cultural y Biológica de los Bosques Pluviales Andinos (DIVA).

Gea-Izquierdo, G., G. M. Pastur, J. M. Cellini, and M. V. Lencinas. 2004. Forty years of silvicultural management in southern Nothofagus pumilio primary forests. Forest Ecology and Management 201:335-347.

Kessler, M. 2002. The "Polylepis problem": where do we stand. Ecotropica 8:97-110.

Kessler, M., J. Böhner, and J. Kluge. 2007. Modelling tree height to assess climatic conditions at tree lines in the Bolivian Andes. Ecological Modelling 207:223-233.

Lavandero, B., A. Labra, C. C. Ramírez, H. M. Niemeyer, and E. Fuentes-Contreras. 2009. Species richness of herbivorous insects on Nothofagus trees in South America and New Zealand: the importance of chemical attributes of the host. Basic and Applied Ecology 10:10-18.

Navarro, G., S. Arrázola, J. A. Balderrama, W. Ferreira, N. De la Barra, C. Antezana, I. Gómez, and M. Mercado. 2013. Diagnóstico del estado de conservación y caracterización de los bosques de Polylepis en Bolivia y su avifauna. Revista Boliviana de Ecología y Conservación Ambiental 28:1-35.

Pickering, C., and J. Byrne. 2014. The benefits of publishing systematic quantitative literature reviews for PhD candidates and other early-career researchers. Higher Education Research and Development 33:534-548.

Pickering, C., J. Grignon, R. Steven, D. Guitart, and J. Byrne. 2014. Publishing not perishing: how research students transition from novice to knowledgeable using systematic quantitative literature reviews. Studies in Higher Education 40:1756-1769.

Pollice, J., P. Marcora, and D. Renison. 2013. Seed production in Polylepis australis (Rosaceae) as influenced by tree size, livestock and interannual climate variations in the mountains of central Argentina. New Forests 44:233-247.

Premoli, A. C., M. C. Acosta, P. Mathiasen, and C. Donoso. 2012. Variación genética en Nothofagus (subgénero Nothofagus). Bosque (Valdivia) 33:115-125.

Rada, F., C. García-Núñez, and S. Rangel. 2009. Low temperature resistance in saplings and ramets of Polylepis sericea in the Venezuelan Andes. Acta Oecologica 35:610-613.

Rada, F., G. Goldstein, A. Azócar, and F. Meinzer. 1985. Daily and seasonal osmotic changes in a tropical treeline species. Journal of Experimental Botany 36:989-1000.

Renison, D., A. M. Cingolani, and R. Suárez. 2002. Efectos del fuego sobre un bosquecillo de Polylepis australis (Rosaceae) en las montañas de Córdoba, Argentina. Revista Chilena de Historia Natural 75:719-727.

Renison, D., G. A. E. Cuyckens, S. Pacheco, G. F. Guzmán, H. R. Grau, P. Marcora, G. Robledo, A. M. Cingolani, J. Domínguez, M. Landi, L. Bellis, and I. Hensen. 2013. Distribución y estado de conservación de las poblaciones de árboles y arbustos del género Polylepis (Rosaceae) en las montañas de Argentina. Ecología Austral 23:27-36.

Renison, D., I. Hensen, and A. M. Cingolani. 2004. Anthropogenic soil degradation affects seed viability in Polylepis australis mountain forests of central Argentina. Forest Ecology and Management 196:327-333.

Renison, D., I. Hensen, and R. Suárez. 2011. Landscape structural complexity of high-mountain Polylepis australis forests: A new aspect of restoration goals. Restoration Ecology 19:390-398.

Renison, D., I. Hensen, R. Suárez, and A. M. Cingolani. 2006. Cover and growth habit of Polylepis woodlands and shrublands in the mountains of central Argentina: human or environmental influence? Journal of Biogeography 33: 876-887.

Renison, D., M. P. Chartier, M. Menghi, P. I. Marcora, R. C. Torres, M. Giorgis, I. Hensen, and A. M. Cingolani. 2015. Spatial variation in tree demography associated to domestic herbivores and topography: Insights from a seeding and planting experiment. Forest Ecology and Management 335:139-146.

Sancho, R., F. Morillo, D. De Filippo, I. Gómez, M. T. Fernández. 2006. Indicadores de colaboración científica intercentros en los países de América Latina. Interciencia 31:284-292

Schmidt-Lebuhn, A. N., M. Kessler, and M. Kumar. 2006. Promiscuity in the Andes: species relationships in Polylepis (Rosaceae, Sanguisorbeae) based on AFLP and morphology. Systematic Botany 31:547-559.

Toivonen, J. M., M. Kessler, K. Ruokolainen, and D. Hertel. 2011. Accessibility predicts structural variation of Andean Polylepis forests. Biodiversity and Conservation 20:1789-1802.

Torres, R. C., D. Renison, I. Hensen, R. Suárez, and L. Enrico. 2008. Polylepis australis' regeneration niche in relation to seed dispersal, site characteristics and livestock density. Forest Ecology and Management 254:255-260.

Underwood, A. J. 1997. Experiments in ecology: their logical design and interpretation using analysis of variance. Cambridge University Press.

Urrego, D. H., B. A. Niccum, C. F. La Drew, M. R. Silman, and M. B. Bush. 2011. Fire and drought as drivers of early Holocene tree line changes in the Peruvian Andes. Journal of Quaternary Science 26:28-36. 
Valencia, B. G., F. Matthews-Bird, D. H. Urrego, J. J. Williams, W. D. Gosling, and M. Bush. 2016. Andean microrefugia: testing the Holocene to predict the Anthropocene. New Phytologist 212:510-522.

Whittaker, R. H. 1975. Communities and ecosystems, 2nd ed. Collier MacMillan, London, UK.

Zimmermann, H., D. Renison, I. Leyer, and I. Hensen. 2009. Do we need livestock grazing to promote Polylepis australis tree recruitment in the Central Argentinean Mountains? Ecological research 24:1075-1081. 